\title{
Is there a link between the prevalence of cardiovascular disease, diabetes, kidney disease, chronic obstructive pulmonary disease and malignancies and the severity of COVID-19 within the BAME population?
}

\author{
Authors: Nuwanji Amarasekera, ${ }^{A}$ Paulomi Sarker ${ }^{A}$ and Saji Manoharan ${ }^{A}$
}

In 2020 the World Health Organization (WHO) declared COVID-19 to be a global pandemic as the disease began to spread across the world. A review by Public Health England confirmed that 'the risk of dying among those diagnosed with COVID-19 is higher in those in BAME groups than in White ethnic groups.' This may be due to both environmental factors and increased genetic susceptibility. Here we look specifically at the disproportionate impact that certain underlying comorbidities may have on the mortality of BAME (Black, Asian, minority ethnic) individuals with COVID-19 infection. We investigated the prevalence of cardiovascular disease, diabetes, renal disease, respiratory conditions and malignancy in the BAME population compared with other ethnicities and also investigated the impact that these underlying conditions may have on the severity of COVID-19 infection. We looked at systematic reviews and original studies along with articles from the BMJ, Lancet, Science Direct, J Am Heart Assoc, WHO database, NHS website, Healthline and BBC News by using key words to search for relevant papers written in English.

Hypertension, diabetes and renal disease were found to be more common in BAME individuals, and respiratory conditions and malignancy were more common in Caucasians. We explored several genetic and some environmental reasons for this difference in prevalence, for example increased sodium retention and sensitivity in Black people, varying endocrine responses to stress, D-dimer levels, fat metabolism rates, industrialisation and slavery. As expected, these underlying comorbidities were often present with a more severe COVID-19 outcome. Finally, we explored why the existence of these conditions may exacerbate infection. Reasons included upregulation and glycosylation of the ACE-2 receptor, increased risk of cardiac complications due to increased oxygen demand, altered host immunity and irregular levels of inflammatory cytokines.

\section{Conflicts of interest}

None declared.

\section{Reference}

1 Siddique H. Key findings from Public Health England's report on Covid-19 deaths. Guardian 20 June 2020. www.theguardian.com/ world/2020/jun/02/key-findings-from-public-health-englandsreport-on-covid-19-deaths [Accessed 02 December 2020].

Author: ${ }^{A}$ Imperial College London, London, UK 